This is good news because commercial producers of word-processing software will then be able to incorporate user-friendly features (tools) permitting the generation of articles with standard SGML tags without an author having detailed knowledge of DTD or SGML. User testing of the SGML-capped programs will probably begin next year and advanced PC's available in five years should be able to handle them. The alternative is to have an author use a standard style-sheet which imposes a structure on a document to permit special translation programs being used by some publishers to process the compuscript. It now seems this route will only be needed for authors who do not possess SGMLcapped word-processing software.

The EPS working group plans to monitor the development of the ISO DTD and to eventually help in its promotion as it is in everyone's interest to have word-processors with

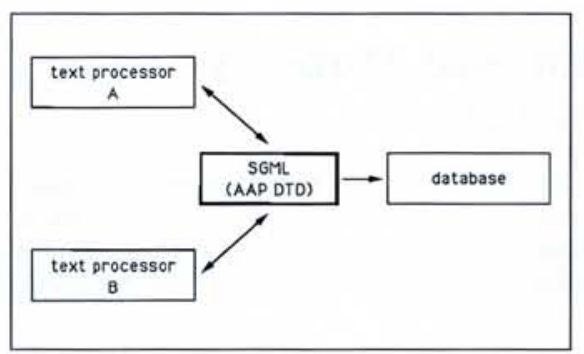

Translation programs to and from SGML are needed so that word-processors can load databases and exchange documents.

SGML capabilities. There remains of course the problem of electronically transmitting figures. Dr. van Herwijnen points out that compression techniques are becoming more economic and widely available. Second, while

\title{
Nuclear Physics Division Board
}

\section{CALL FOR NOMINATIONS}

Members of the Nuclear Physics Division are invited to submit by mid-October 1992 the names of candidates for election to the Divisional Board. Nominations should be sent to Prof. R.M. Bock, GSI-Ges. für Schwerionenforschung, Postfach 1105 52, W-6100 Darmstadt (fax: $+49-6151-359989$ ). Three supporting signatures of IOM's of EPS are required as well as the candidate's written agreement that he or she is willing to serve.

Of the present Board, R. Bergere (Saclay), R.M. Bock (Darmstadt), P. Glaudemans (Utrecht), G. Morrison (Birmingham), and G. Goldring (Rehovot) have served for two four-year terms and cannot stand for re-election. The Board proposes the following for election or reelection $(*)$ :

A. Bertin, Bologna*

H.P. Blok, Amsterdam

J. Durell, Manchester

I. Lovas, Budapest (co-opted)*

W. Nazarewich, Warsaw (co-opted)

Yu. Oganessian, Dubna (co-opted)*

The CNRS, France, and the German Physical Society have each been asked to make one nomination.

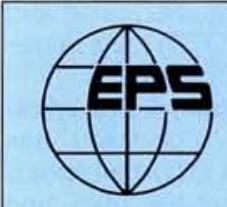

\section{EPS CONDENSED MATTER DIVISION 13th General Conference}

\author{
29 March - 2 April 1993 \\ Regensburg, Bayern, Germany (University of Regensburg)
}

Held in conjunction with the Frahjahrstagung des Arbeitskreises Festkörperphysik

der Deutsche Physikalische Gesellschaft (DPG)

Aims to provide a forum for both senior and junior scientists from throughout Europe to present and discuss their research activities.

Ph.D. students who are active and successful in condensed matter research should be encouraged to participate so as to intensify personal relations within Europe through the presentation and discussion of their scientific results and ideas.

Plenary talks, invited talks, symposia, contributed talks, and posters within each of nine Divisions covering the whole of condensed matter physics:
liquids
low-temperature physics
macromolecular physics
magnetism
metals

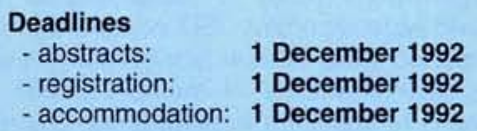

semiconductors and in

surfaces, interfaces and thin films

dynamics, statistical physics and neutron scattering chemical physics
Registration Fees
- individual members of EPS - students:

DPG and national societies: DM 200.

DM 90.-

Further information and registration forms are detailed in the second circular, available from: Prof. Dr. H. Hoffmann, Local Chairman, 13th CMD General Conference

Institut für Angewandte Physik, Universität Regensburg,

Postfach 1010 42, W-8400 Regensburg a Standard Page Description Language (SPDL) is perhaps a generation away, PostScript file structures are effectively a de facto standard. SGML and PostScript mix well so together they could provided an interim, allembracing standard.

\section{- Three Months to Freeze ITER Design Objectives}

Euratom, the US, Japan, and Russia signed a six-year agreement in Washington last month, some 18 months behind schedule, for an Engineering Design Activities (EDA) phase as the basis for "future decisions" on a 5800 M\$US next-generation fusion test reactor ITER that would demonstrate "controlled ignition and extended burn of D-T plasmas, with steady state as the ultimate goal, by demonstrating technologies..." The EDA (costing 1000 MSUS not including physics and some long-term R\&D covered by national fusion programmes) will be implemented by two or more Protocols. The first establishes working groups to review (and presumably freeze) technical objectives within three months, and to draw up work assignments and a draft of a second Protocol for the bulk of the EDA. Three Joint Work Sites (San Diego, USA; Naka, J; and the MPI für Plasmaphysik, Garching, G, responsible for the reactor vessel and contents) can now begin hiring staff (up to about 180 professionals). A Joint Central Team with four Deputy Directors (a principal deputy and the site heads) is divided between the sites and staffed equally by the four partners. It assists the Director, P.-H. Rebut, formerly the JET Undertaking Director, who reports to the ITER Council, which meets twice each year and is assisted by technical and management Advisory Committees. Home Teams reporting to the Director are responsible for the partners' work contributions. Euratom's team led by R. Tosci from Garching is based on the Next European Torus team formed in 1983 , but we shall have to wait until Protocol 2 to know its size and degree of centralisation. Euratom's Fusion Technology Steering Committee - Programmatic (FTSC-P) chaired by $R$. Aymar from the CEA, France, is responsible for deciding European ITER implementation. It reports to the government-level Consultative Committee for the Fusion Programme (CCFP) which is seeing its power weakened as the EC Framework programme structure evolves.

In the words of European experts, "the price of international cooperation is the impression of a snail's pace with Europe losing its lead". Difficult choices must also be faced. Japan favours an all-encompassing (and expensive) ITER device; Europe a slimmed down version with much covered by national programmes. Some argue for pulse operation; the US is more interested in steady state. A large reactor gives a margin for confinement; a smaller device may not produce ignition. Dr. Rebut hopes that the all-important site selection is made within a year or two as much engineering design is site-dependent (using a green-field site would be a "nonsense" as existing investments must be exploited). But the greatest challenge facing the plasma community is perhaps the change in culture: few of Europe's more experienced plasma physicists will be involved in ITER when it starts operation in the year 2005 at the earliest. 